\title{
Cohort study on educational well-being of children of Chinese \\ origin adopted into transracial and international families in
}

\section{the Spanish education system [version 1; peer review: 1}

\section{approved, 1 approved with reservations]}

\author{
David Doncel-Abad (Di), Pablo Cabrera-Álvarez² \\ ${ }^{1}$ Sociology and Communication, University Salamanca, Salamanca, 37007, Spain \\ ${ }^{2}$ Sociology and Communication, University of Salamanca, Salamanca, 37007, Spain
}

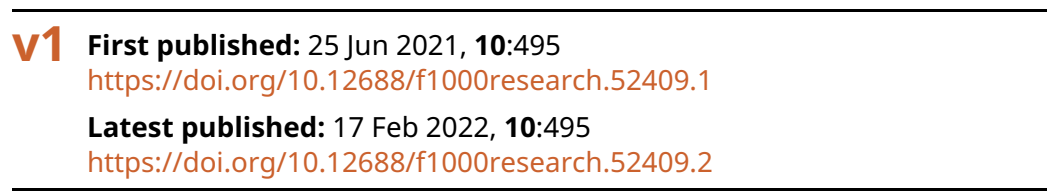

\section{Abstract}

The following dataset focuses on the educational well-being of adopted girls of Chinese origin in Spanish schools. Due to its characteristics, the presence of this group may generate complex interaction dynamics in school; particularly regarding bullying in school linked to factors such as the acceptance of others. These are dynamics, which may indeed condition the educational experience of this social group. Therefore, the aim of creating this dataset is to measure the educational well-being of children of Chinese origin adopted into transracial families in Spain. Although this research was justified by the lack of studies on this social group, we aimed to go one-step further, we also studied the correlation between this social group's educational experience, and to what extent they show an interest in Chinese culture. As we have written before, we incorporated the concept of Well-Being and we worked with the following indicators: Satisfaction with Life, Social Life, and Bullying in School, Racial Bullying, Personal Identity and Interest in Chinese Culture. To achieve the objective set forth, we conducted a questionnaire. The final sample consisted of 268 individuals. The creation of this dataset provided us with information that can shed light on the relationship between adoption, race/ethnicity and the educational experience of adopted children of Chinese origin.

Keywords

Well-being, transnational adoption, race and ethnicity

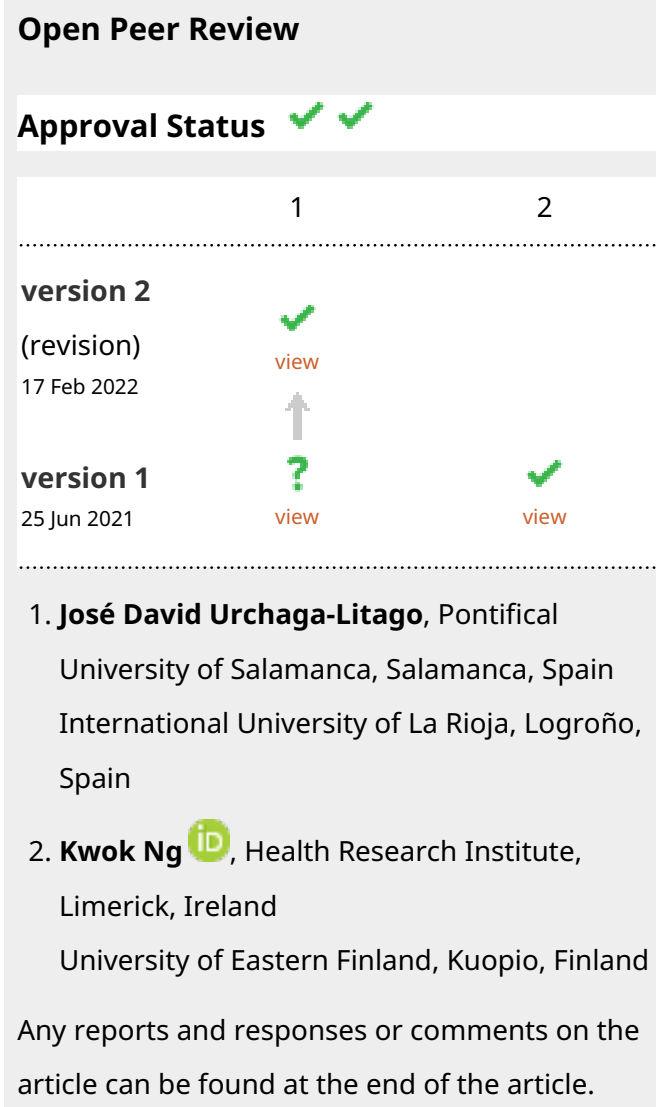

1 2

version 2

(revision)

17 Feb 2022

version 1

25 Jun 2021

1. José David Urchaga-Litago, Pontifical University of Salamanca, Salamanca, Spain International University of La Rioja, Logroño, Spain

2. Kwok Ng iD, Health Research Institute, Limerick, Ireland University of Eastern Finland, Kuopio, Finland Any reports and responses or comments on the article can be found at the end of the article. 


\section{Corresponding author: David Doncel-Abad (davidoncel@usal.es)}

Author roles: Doncel-Abad D: Conceptualization, Data Curation, Funding Acquisition, Investigation, Methodology, Project Administration, Resources, Supervision, Validation, Writing - Original Draft Preparation, Writing - Review \& Editing; Cabrera-Álvarez P: Data Curation, Formal Analysis, Methodology, Software, Validation, Visualization, Writing - Original Draft Preparation, Writing - Review \& Editing

Competing interests: No competing interests were disclosed.

Grant information: This work was supported by Government of Castilla y León: SA157G18 (J425-463AC03).

The funders had no role in study design, data collection and analysis, decision to publish, or preparation of the manuscript.

Copyright: (C) 2021 Doncel-Abad D and Cabrera-Álvarez P. This is an open access article distributed under the terms of the Creative Commons Attribution License, which permits unrestricted use, distribution, and reproduction in any medium, provided the original work is properly cited.

How to cite this article: Doncel-Abad D and Cabrera-Álvarez P. Cohort study on educational well-being of children of Chinese origin adopted into transracial and international families in the Spanish education system [version 1; peer review: 1 approved, 1

approved with reservations] F1000Research 2021, 10:495 https://doi.org/10.12688/f1000research.52409.1

First published: 25 Jun 2021, 10:495 https://doi.org/10.12688/f1000research.52409.1 


\section{Introduction}

The following study focuses on the social well-being or educational experience of adopted girls of Chinese origin in Spanish schools. At the end of the 1990s, Spain became the world's largest adoption receiving country in the world. Geographically, Asia, and specifically China, was the prefered place among adoptive parents. According to data from the National Statistics Institute of Spain, the first girls adopted from China began to arrive in 1995. From that moment on, Chinese children soon became the most adopted of all: in 2005, out of 2,854 adopted children of Asian origin, 2,753 came from China. A decade later, it is estimated that there are approximately 18,000 adopted children of Chinese origin in Spain (around 400 in Castilla y León), which makes this the largest group when it comes to international adoptions.

This social group presents a characteristic, which is typical of adoptive families who are of dual nationality and ethnicity: on the one hand, they have Asian phenotypic traits from birth; yet on the other, they are part of the dominant culture due to adoption (Grotevant \& VonKorff, 2011). This characteristic, named "the transracial paradox" by Lee (2003), has contributed to the enrichment of social heterogeneity in the classrooms but has also increased the complexity of the relationships formed in the schools (Kim \& Lee, 2020). We must not forget that a key element to achieving a necessary and proper coexistence lies in the development of the relationships between the different members of the educational community.

Scientific literature on this matter has indicated that one factor linked to bullying in school is racial/ethnic prejudice (Verkuyten \& Thijs 2001, 2002, 2006). In particular, a correlation between Asian race or ethnicity and bullying in school has been identified by Juvonen, Grahan and Schsuter (2003) and Mouttapa, Valente, Gallaher, Rohrbach and Unger (2004). Admittedly, it is also indicated that racial/ethnic factor alone does not increase the likelihood of being victimized (Díaz Aguado et al., 2013), yet it is an influencing factor when linked to an array of material and/or social circumstances.

Few studies have been carried out on the analysis of racial/ethnic bullying in school together with the adoption factor in the case of transracial adoptive families. However, there have been significant contributions. Raaska et al. (2012) found that being adopted increases the chances of being bullied in school, but only when combined with other individual variables. Moran et al. (1993) proved that the "being Asian" factor has an impact when it comes to friendship or companionship compared to other social groups. As for the studies carried out by Adams, Tessler and Gamache (2005) and Tang and Arthur (2012), it was proven that if an educational environment offers a positive experience to adopted individuals belonging to transracial families, this contributes to the child's positive acceptance of the uniqueness of their ethnic or racial origin, fosters their self-esteem and, consequently, their educational well-being. In Spain, Fernández (2016) pointed out that "being Chinese and adopted becomes 'something bad' and leads to insults or even bullying" (Fernández-Cáceres, 2016, p. 409). However, Gil, Doncel, Morales and Lambea (2020) revealed that these children may be mocked due to their Asian phenotypic traits, yet not for being adopted.

In summary, the group of children of Chinese origin adopted in Spain can be considered a group whose presence in schools may generate complex interactional dynamics. Particularly regarding bullying in school linked to factors such as the acceptance of the "other". These are dynamics, which may indeed condition the educational well-being of this social group.

Consequently, this study aimed to create a dataset that presented the educational well-being of adopted children of Chinese origin in transracial families in Spain, placing special focus on Castilla y León. Although this research was justified by the lack of studies on this social group, we aimed to go one-step further and we also studied the correlation between this social group's educational experience and to what extent they show an interest in Chinese culture.

\section{Methods}

\section{Framework}

The initial target population of study was formed of female teenagers adopted from the People's Republic of China who were enrolled in Castilla y León schools. The initial age range was between 14 and 16 years (both ages included). All these girls must have arrived in Spain around 2003-2005, as the data collection started on the 2019-2020 school year.

According to the data offered by the Directorate-General for Social Policy, Families and Childhood, under the Ministry of Health, Social Policy and Equality (2010, p. 108), there were a total of 7,612 adoptions from China between 2003 and 2005, which makes this country the most notable when it comes to adoption numbers. Also, taking into account the fact that most adopted children are girls (around 97\% according to the data from Castilla y León; Fernández Cáceres, 2016; SSCYL, 2018), we could estimate that the number of girls of Chinese origin was around 7,380. The census carried out between 1997 (the year when the city of Burgos started receiving the first girls from China) and January of 2020 registered a total of 820 adoptions in Castilla y León. Out of these 820 adoptions made in the People's Republic of China, 800 were 
girls (97.6\%), and 20 were boys (2.4\%) (SSCYL, 2018; Fernández Cáceres, 2016). The strict birth control measures and the one-child policy implemented in the People's Republic of China, together with the patriarchal tradition of preferring sons to daughters, explains why girls were abandoned more than boys.

\section{Sampling procedure}

As we were lacking a sampling framework, we decided to ask for cooperation from adoptive parents' associations to be able to access the target population. We collected data through a census, which showed parents who had joined adoption associations and who were able to complete an adoption process in China between 2003 and 2006. This approach is justified by the impracticability of screening the general population in order to locate homes with adopted children. Furthermore, this strategy is based on the evidence that a large number of parents who adopt children from China tend to join an association in order to go through this process with greater guarantees.

This strategy allowed us to access a large group of adopted children through their parents. In order to do so, we created an exhaustive list of adoptive parents' associations registered in Spain: ADOPCHINA, AFADA, AFADENA, AFAAR, AFAHU, AFAIC, AFAMUNDI, AIBA, AMADA, ANICHI, ARFACYL, ASTURADOP, ATLAS, AKUNA INTERNACIONAL, ACI, Hijos que esperan, MANANIA, MUDAN, UME ALAIA, TRANSMES, Fundación CORA and AFAC. The research team requested these associations to forward our study to their members who met the requirements to be a part of the target population for this research.

The online questionnaire addressed to adopted children was sent by email to the contacts provided. We first sent the questionnaire on September 12, 2019 at 12:00 AM. The responses to the questionnaire were gathered over three months (from October 2 to December 17). While compiling the responses, we exhaustively monitored the sample and the established exclusion criteria.

The incidents we encountered while gathering the responses to the questionnaire were as follows:

- A questionnaire was deleted because it was duplicated.

- Two questionnaires were deleted because they were incomplete.

On December 17, we obtained the final sample, which consisted of $n=268$ individuals between 9 and 19 years, both ages included. Of them, $98.1 \%$ were girls and $1.9 \%$ boys.

It is worth noting that there could have been some measurement bias due to the children filling in the questionnaire under their parents' supervision. It is possible that the children felt inhibited or that their responses were not as precise because of their parents being present.

\section{Variables}

In this study, we adopted the concept of Well-Being used by the Organization for Economic Cooperation and Development (OECD) in its PISA 2017 report in order to measure the educational well-being of this social group in schools. The OECD related the concept of students' social well-being to a variety of factors: satisfaction with life, schoolwork-related anxiety (homework and tests), motivation to achieve, sense of belonging at school, bullying, and social status and inequalities. Taking into account these elements influencing the concept of Educational Well-being, and in accordance with the purpose of this study, we have looked at the following factors: Satisfaction with Life, Social Life, Bullying in School, Racial/Ethnic Bullying, Personal Identity and Interest in Chinese Culture.

- Satisfaction with Life measures the degree of subjective well-being of students in relation to their own lives. In the questionnaire used in this study, the interviewees' satisfaction was rated on a scale of 0 to 10,0 being the lowest satisfaction and 10 the highest.

- Social Life $(\alpha=.81)$ has been defined as social integration or social relationships that the individual has established with his or her classmates. The items used to measure the degree of social integration were: "We get along well in the classroom," "I express and defend my opinions without hurting others," "I feel that I have friends," "My classmates care about me when I need them," "I like to collaborate with my friends," "My classmates like me," "I'd rather work in teams than alone," "I felt different from the rest," "It is hard for me to express my feelings." Each indicator was rated on a Likert scale, ranging from 1 (Strongly Disagree) to 5 (Strongly Agree). Based on these indicators, we created the Social Life Scale, the scale being from 1 (low degree of social integration at school) to 5 (high degree of social integration). 
- Bullying ( $\alpha=.72$ ) has been defined as "an intentional, repeated, negative (unpleasant or hurtful) behavior by one or more persons directed against a person who has difficulty defending himself or herself” (Olweus, 2006, p. 81). In this research, this scope was studied by asking the typical questions to measure bullying in PISA: "I have been physically hurt by other students," "Other students took my things from me," "Other students insulted me/called me names," "Other students lied about me, talked behind my back or spread rumors about me," "I was threatened by other students," "Other students left me out of things/isolated me on purpose," "Other students destroyed my things" and "Other students made fun of me." Each indicator was measured on a Likert scale (1-Never, 2-Rarely, 3-Sometimes, 4-Frequently and 5-Always). From these indicators, we created the Bullying Scale, ranging from 1 (low perception of having experienced bullying) to 5 (high perception of having experienced bullying).

- Racial/Ethnic Bullying was studied by adding a racial element to the typical questions to measure bullying: "I felt intimidated, rejected or bullied because of my facial features." This indicator was measured on a Likert scale (1-Never, 2-Rarely, 3-Sometimes, 4-Frequently and 5-Always).

- Personal Identity: In order to measure children's perception of identity, we used one of the Social Life indicators: "I felt different from others." This item was measured on a Likert scale, 1 being Strongly Disagree (greater integration) and 5 being Strongly Agree (little integration).

- Interest in Chinese Culture $(\alpha=.88)$, measured by the following indicators: "News about China interests me," "I'd like to learn Chinese," "I'd like my parents to go to Chinese restaurants more often," "I'd like to attend Chinese celebrations," "I'd like to know how non-adopted Chinese children live in my town," "I'd like my parents to take me to China" and "I'd like my school to organize activities so I can learn about Chinese culture." Each item was measured on a Likert scale, 1 being Strongly Disagree and 5 Strongly Agree. Based on these indicators, we created the Interest in Chinese Culture Scale, the scale ranging from 1 (little interest in learning about Chinese culture) to 5 (strong interest).

We added a set of sociodemographic variables to the analysis, these being:

- ISCED: Scale to measure the parents' highest completed level of education, 1 being completed secondary education or lower and 5 being university education (master's degree or doctorate).

- Age: recoded in four intervals: $9-13 ; 14-15 ; 16-17$ and $18-19$ years old.

- Age of arrival in Spain, measured in months.

- Type of school: Público (Public School), Concertado (Charter School), Privado (Private School)

- Sex.

\section{Composition of the final dataset}

The final sample $(n=268)$ consisted of a larger number of participants than expected from the initial sample design. Also, a broader part of the Spanish territory was represented. However, the sample criteria established for Castilla y León, which was the region we were interested in, were not met. We obtained data from adopted children of Chinese origin from the following Autonomous Communities (see Table 1):

In the following table (Table 2), we can observe the profile of the final sample by sociodemographic variables:

\section{Summary}

The main results obtained show:

- The incidence of bullying in school is similar to the parameters observed in other reports (like PISA 2015) concerning the school population in Spain.

- Moreover, this group stands out in particular due to its high level of Satisfaction with Life (in relation to expected results by age group compared to data from the PISA 2015 report for Spain) and its low perception of feeling different from others. 
Table 1. Distribution of the sample in autonomous communities in Spain.

\begin{tabular}{|l|l|l|l|l|}
\hline \multirow{2}{*}{ Valid } & Frequency & Valid percentage & Cumulative percentage \\
\cline { 2 - 4 } & Andalucía & 30 & 11.2 & 11.2 \\
\hline Aragón & 13 & 4.9 & 16.0 \\
\hline Canarias & 2 & .7 & 16.8 \\
\hline Cantabria & 7 & 2.6 & 19.4 \\
\hline Castilla La-Mancha & 19 & 7.1 & 26.5 \\
\hline Castilla y León & 72 & 26.9 & 53.4 \\
\hline Cataluña & 12 & 4.5 & 57.8 \\
\hline Comunidad de Madrid & 48 & 17.9 & 75.7 \\
\hline Comunidad Foral de Navarra & 3 & 1.1 & 76.9 \\
\hline Comunidad Valenciana & 9 & 3.4 & 80.2 \\
\hline Extremadura & 17 & 6.3 & 86.6 \\
\hline Galicia & 15 & 5.6 & 92.2 \\
\hline Islas Baleares & 2 & .7 & 92.9 \\
\hline La Rioja & 7 & 2.6 & 95.5 \\
\hline País Vasco & 5 & 1.9 & 97.4 \\
\hline Principado de Asturias & 7 & 2.6 & 100.0 \\
\hline Total & 268 & 100.0 & \\
\hline
\end{tabular}

Table 2. Sample profile by sociodemographic variables.

\begin{tabular}{|c|c|c|c|}
\hline & & Cases & $\%$ \\
\hline \multirow[t]{2}{*}{ Sex } & Male & 5 & 1.9 \\
\hline & Female & 263 & 98.1 \\
\hline Age of arrival in Spain & $\begin{array}{l}\text { Less than } 1 \text { year old } \\
\text { From } 1 \text { to } 2 \text { years old } \\
2 \text { years old or more }\end{array}$ & $\begin{array}{l}72 \\
135 \\
61\end{array}$ & $\begin{array}{l}26.9 \\
50.4 \\
22.8\end{array}$ \\
\hline \multirow[t]{4}{*}{ Current school level } & $\begin{array}{l}\text { Primaria (Elementary Education) } \\
\text { Secundaria (Middle School) }\end{array}$ & $\begin{array}{l}4 \\
123\end{array}$ & $\begin{array}{l}1.5 \\
46.1\end{array}$ \\
\hline & Bachillerato (High School) & 103 & 38.6 \\
\hline & Ciclos formativos (Vocational Education) & 20 & 7.5 \\
\hline & Universidad (University) & 17 & 6.4 \\
\hline Type of school & $\begin{array}{l}\text { Público (Public School) } \\
\text { Concertado (Charter School) } \\
\text { Privado (Private School) }\end{array}$ & $\begin{array}{l}188 \\
62 \\
18\end{array}$ & $\begin{array}{l}70.1 \\
23.1 \\
6.7\end{array}$ \\
\hline \multirow[t]{5}{*}{$\begin{array}{l}\text { Parents' highest completed level of } \\
\text { education }\end{array}$} & $\begin{array}{l}\text { Secundarios o menos (Middle School or lower) } \\
\text { FP Grado medio (Vocational Ed.: medium-level) }\end{array}$ & $\begin{array}{l}11 \\
15\end{array}$ & $\begin{array}{l}4.2 \\
5.7\end{array}$ \\
\hline & Bachillerato (High School) & 26 & 9.8 \\
\hline & FP Grado Superior (Vocational Ed.: higher-level) & 14 & 5.3 \\
\hline & $\begin{array}{l}\text { Universitarios - primer y segundo ciclo (Univ.: } \\
\text { Bachellor's) }\end{array}$ & 168 & 63.6 \\
\hline & $\begin{array}{l}\text { Universitarios - máster y doctorado (Univ.: } \\
\text { Master's/PhD) }\end{array}$ & 30 & 11.4 \\
\hline
\end{tabular}


- The fact that there was not a defined sampling framework that the researchers could access led them to use associations as intermediary actors in order to distribute the questionnaire among the target population of study. This lack of a sampling framework implied that many adopted children meeting the criteria for the target population were not included in the sample because their parents did not belong to an adoption association.

- Due to the difficulties in accessing such a specific population, we created a census from the lists of members of the Spanish associations of parents who adopted their children in China. When generalizing the results, we must take into account that not all adoptive parents are part of an association and that some of the contacted parents declined to respond. These two sources of bias (coverage and nonresponse) are common to most surveys.

\section{Ethical approval}

Since the sample would be partially made up of minors, we requested the approval of the Bioethics Committee from the University of Salamanca. After checking that an ethical collection of minors data was guaranteed, the Bioethics Committee approved this project (ID: 390) on September 30, 2019.

\section{Consent}

To carry out this project, before collecting the questionnaires, we obtained the informed consent form prior to the participants or their parents, in cases of minors, taking part in the research.

\section{Data availability}

The data collected for this study cannot be made available to the public in an open repository since the parents, who consented before the children responded to the questionnaire, were informed that the data would be encoded, anonymized and stored in the Archives of the Faculty of Social Science of Salamanca-and that they would only be used for the purposes of this project. It was also agreed that, if any other researchers wished to access this database, they would have to contact the Faculty of Social Science of the University of Salamanca.

This project's database is stored in the Archives of the Faculty of Social Science of the University of Salamanca, located in Campus Unamuno s/n, Salamanca 37006, Spain. This space can only be accessed by the members of the Deanery and the University Secretariat. If a third party wished to access this material, they would need to ask the Archives authorities for the corresponding authorization. Contact details: davidoncel@usal.es Telephone 923294500 Ext. 3113. All the information derived from this study will be used exclusively for the purposes indicated in the initial project.

\section{Acknowledgements}

This database is part of the Research Project SA157G18 (J425-463AC03), entitled "Antropología transversal del conocimiento: Castilla-León y Asia Oriental", developed by the accredited research group Humanismo Eurasia from the University of Salamanca. Funding Entity: Junta de Castilla y León. This article is based on a previous study entitled "Study on Child Well-being of Adopted Chinese Children at Schools in Castilla y León". Source: Pedagogía Social. Revista Interuniversitaria, Issue 35, p. 81-93. DOI:10.7179/PSRI_2019.35.06. It is also based on the article "Educational Well-being and Social Identity of Children of Chinese Origin Adopted in Spain", which is currently being reviewed by the Adoption Quarterly journal.

Díaz-Aguado J, Martínez Arias R, Martín Babarro J: El acoso entre adolescentes en España. Prevalencia, papeles adoptados por todo el grupo y características a las que atribuyen la victimización: Bullying among adolescents in Spain. Prevalence, participants' roles and characteristics attributable to victimization by victims and aggressors. Ministerio de Educación; 2013.

Fernández Cáceres MI: Familias castellanoleonesas adoptantes en China, 1995-2015: decisión de adoptar, relaciones familiares y estrategias de conciliación. Tesis Doctoral. Salamanca: Universidad de Salamanca; 2016.

Publisher Full Text

Gil Villa F, Doncel Abad D, Morales Romo N, et al.: Percepción del bienestar social de niños y niñas adoptadas de origen chino en las escuelas en Castilla y León. Pedagogía social: revista interuniversitaria. 2020; 35: 73-85.

Publisher Full Text
Grotevant HD, Von Korff L: Adoptive identity. In:Handbook of identity theory and research. New York, NY: Springer; 2011; (pp. 585-601).

Juvonen J, Graham S, Schuster MA: Bullying among young adolescents: the strong, the weak, and the troubled. Pediatrics. 2003 Dec; 112(6 Pt 1): $1231-7$

PubMed Abstract | Publisher Full Text

Kim AY, Lee RM: A critical adoption studies and Asian americanist integrative perspective on the psychology of Korean adoption. In: Miller G, Helder E, Marr E. (editors) The Routledge Handbook of Adoption, Routledge International Handbooks. 2020; pp. 120-134

Lee RM: The transracial adoption paradox: History, research, and counseling implications of cultural socialization. Couns Psychol. 2003; 31(6): 711-744.

PubMed Abstract | Publisher Full Text | Free Full Text 
Ministerio del Interior: Sistema Estadístico de Criminalidad. 2016 Reference Source

Moran S, Smith PK, Thompson D, et al.: Ethnic differences in experiences of bullying: Asian and white children. British J Edu Psychol. 1993; 63(3): 431-440.

Publisher Full Text

Mouttapa M, Valente T, Gallaher P, et al.: Social network predictors of bullying and victimization. Adolescence. 2004; 39(154), 315.

PubMed Abstract

OECD:PISA 2015 Results (Volume III): Student's Well-Being. ParísOECD; 2017.

Raaska H, Lapinleimu H, Sinkkonen J, et al.: Experiences of school bullying among internationally adopted children: Results from the Finnish Adoption (FINADO) Study. Child Psychiatry Hum Dev. 2012; 43(4), 592-611.

PubMed Abstract | Publisher Full Text

Tan TX, Jordan-Arthur B: Adopted Chinese girls come of age: Feelings about adoption, ethnic identity, academic functioning, and global self-esteem. Children and Youth Services Review. 2012; 34(8): 1500-1508. Publisher Full Text
Thomas KA, Tessler RC: Bicultural socialization among adoptive families: Where there is a will, there is a way. J Family Issues. 2007; 28(9): 1189-1219.

Publisher Full Text

SSCYL (Servicios Sociales de Castilla y León): Boletines informativos anuales sobre adopción. 1999-2018

Reference Source consulta en junio de 2018.

Verkuyten $\mathrm{M}$, Thijs J: Peer victimization and self-esteem of ethnic minority group children. J Community y Appl Soc Psychol. 2001; 11(3): 227-234.

Publisher Full Text

Verkuyten M, Thijs J: School satisfaction of elementary school children: The role of performance, peer relations, ethnicity and gender. Soc Indicators Res. 2002; 59(2): 203-228.

Publisher Full Text

Verkuyten M, Thijs J: Ethnic discrimination and global self-worth in early adolescents: The mediating role of ethnic self-esteem. Int J Behav. Develop. 2006; 30(2): 107-116.

Publisher Full Text 


\title{
Open Peer Review
}

\section{Current Peer Review Status:}

\section{Version 1}

Reviewer Report 11 February 2022

https://doi.org/10.5256/f1000research.55683.r100286

(C) $2022 \mathrm{Ng} \mathrm{K}$. This is an open access peer review report distributed under the terms of the Creative Commons Attribution License, which permits unrestricted use, distribution, and reproduction in any medium, provided the original work is properly cited.

\author{
Kwok Ng \\ 1 Physical Activity for Health, Health Research Institute, Limerick, Ireland \\ 2 University of Eastern Finland, Kuopio, Finland
}

This is a nicely reported data set with rationale and background to the sample. Furthermore, given the limitations of the sampling frame, the authors have done a good job to locate such a diverse range of participants with similar characteristics of interest.

The data availability is of course a limitation, but the authors have given information for how to retrieve it, and although it has not been attempted, it is hoped that it would not impede on the progress of open science.

The variables are slightly limited, given the efforts taken to collect data from the sample, and a more holistic perspective of health may have been warranted. None the less, this is a starting point to understand the exposure to victimisation and self-perceptions around this matter from the perspective of Chinese adopted girls in Spain.

Is the rationale for creating the dataset(s) clearly described?

Yes

Are the protocols appropriate and is the work technically sound?

Yes

Are sufficient details of methods and materials provided to allow replication by others? Yes

Are the datasets clearly presented in a useable and accessible format? Partly

Competing Interests: No competing interests were disclosed.

Reviewer Expertise: public health and health promotion. 


\section{I confirm that I have read this submission and believe that I have an appropriate level of expertise to confirm that it is of an acceptable scientific standard.}

Reviewer Report 29 November 2021

https://doi.org/10.5256/f1000research.55683.r100287

(c) 2021 Urchaga-Litago J. This is an open access peer review report distributed under the terms of the Creative Commons Attribution License, which permits unrestricted use, distribution, and reproduction in any medium, provided the original work is properly cited.

José David Urchaga-Litago

${ }^{1}$ Faculty of Communication, Pontifical University of Salamanca, Salamanca, Spain

2 Faculty of Psychology, International University of La Rioja, Logroño, Spain

Abstract: Studies the incidence of bullying in school and satisfaction with life in a sample of children adopted from China.

This study is very new in Spain. In addition, it is very important because adopted children of Chinese origin is the largest group.

It is original to study "to what extent they show interest in Chinese culture", but it is important to justify why that is, to briefly justify its theoretical importance. Neither is any result provided on this aspect. In this case, either present results or eliminate said objective (I recommend the latter).

Well defined population and sample. Well measured variables. The authors are requested to reference the original studies of the measuring instruments. If they have been created "ad hoc" for this research, the authors must indicate it.

Table 1: Eliminate the accumulated percentage.

Important: There is no Results section. Provide the study data about these conclusions:

1. The incidence of bullying in school is similar to the parameters observed in other reports (like PISA 2015) concerning the school population in Spain.

2. Moreover, this group stands out in particular due to its high level of satisfaction with life (in relation to expected results by age group compared to data from the PISA 2015 report for Spain) and its low perception of feeling different from others.

I do not know if the journal admits publishing studies without results, only presenting the conclusions. If so, I apologize for the last entry.

I consider this to be a very important and original study. I think it should be indexed.

Is the rationale for creating the dataset(s) clearly described? 
Yes

Are the protocols appropriate and is the work technically sound?

Yes

Are sufficient details of methods and materials provided to allow replication by others? Yes

Are the datasets clearly presented in a useable and accessible format?

Partly

Competing Interests: No competing interests were disclosed.

Reviewer Expertise: Methodology and Statistics in Social and Human Sciences

I confirm that I have read this submission and believe that I have an appropriate level of expertise to confirm that it is of an acceptable scientific standard, however I have significant reservations, as outlined above.

Author Response 15 Feb 2022

David Doncel-Abad, University Salamanca, Salamanca, Spain

First, we want to thank the reviewer for his useful comments that have been fundamental to refining the final version of the paper. Moreover, we would like to add some clarifications to some of your questions and highlight the changes introduced in the new version of the paper.

We adapted the statement about the research objectives, which are broader than assessing to what extent "the Chinese adopted children show interest in Chinese culture". This secondary objective will be developed and assessed in other publications in the frame of the research project. We understand that this statement could be misleading, and we have removed it from the paper.

We have included the references of the measures used in the study.

Regarding the absence of a results section: This is a data note in which we describe a dataset and inform about the conditions under which other researchers can access it. The substantive results of this research are presented in other papers that are under review at the moment. However, we agree with the reviewer that the comments about the conclusions should not be part of a data note, and, therefore, we have removed them from the paper.

We improved the paper with further clarifications in the methods section and style changes.

Competing Interests: No competing interests were disclosed. 
The benefits of publishing with F1000Research:

- Your article is published within days, with no editorial bias

- You can publish traditional articles, null/negative results, case reports, data notes and more

- The peer review process is transparent and collaborative

- Your article is indexed in PubMed after passing peer review

- Dedicated customer support at every stage

For pre-submission enquiries, contact research@f1000.com 